\title{
THE BALTIC STATES AFTER THE COLLAPSE OF THE SOVIET UNION: APPENDIX
}

\author{
TOIVO U. RAUN \\ Indiana University, Bloomington, IND, \\ USA
}

Two population tables, which were to have accompanied my article in the previous issue of Hungarian Studies (14/2 [2000], 275-284), were inadvertently omitted. They are published here as an appendix to that text.

Tables 1 and 2 provide an overview of the evolution of the ethnic composition of Estonia, Latvia, and Lithuania since the interwar era. They indicate a notable divergence in demographic trends in Lithuania in comparison with the other two Baltic states. Despite suffering the same kinds of population losses in World War II and under Stalinism as the Estonians and Latvians, the Lithuanians displayed a strong demographic dynamism, based on higher birth rates, and maintained a remarkably stable share of the total population of their country. On the other hand, demographic growth in Estonia and Latvia had already slowed considerably by the interwar period, and the native population in those two countries was much less able to withstand the disasters of the 1940s. It is striking that in 1989 there were fewer Estonians in Estonia and Latvians in Latvia than in the 1930s. It is also noteworthy that the number of ethnic Russians in Latvia throughout the Soviet era was more than double the combined corresponding figure for Estonia and Lithuania, a phenomenon that reflected Riga's attractiveness and size as the one true metropolis in the Baltic states.

During the 1990s, Estonia and Latvia witnessed an immediate population decline, caused mainly by initially high levels of out-migration, while Lithuania managed to sustain some demographic growth until 1993. Nevertheless, the native proportion of the population in Estonia and Latvia gradually increased during this decade, mainly because Estonians and Latvians were less likely to emigrate permanently than Russians and other non-Balts. By the second half of the 1990s, demographic trends in Lithuania finally began to converge with those that had prevailed earlier in Estonia and Latvia, especially with regard to a negative natural increase and a decreasing total population, although the rate of decline remained slower in the Lithuanian case.

Hungarian Studies 15/1 (2001)

0236-6568/2001/\$5.00 @ 2001 Akadémiai Kiadó, Budapest 
Table 1. Ethnic composition of the Baltic States (in 1,000's)

ESTONIA

\begin{tabular}{|c|c|c|c|c|c|c|}
\hline & $1934^{\mathrm{a}}$ & 1959 & 1970 & 1979 & 1989 & $1999^{b}$ \\
\hline Estonians & 993.5 & 892.7 & 925.1 & 947.8 & 963.3 & 942.5 \\
\hline Russians & 92.7 & 240.2 & 334.6 & 408.8 & 474.8 & 406.0 \\
\hline Ukrainians & - & 15.8 & 28.1 & 36.0 & 48.3 & 36.7 \\
\hline Belorussians & - & 10.9 & 18.7 & 23.5 & 27.7 & 21.4 \\
\hline Finns & 1.1 & 16.7 & 18.5 & 17.6 & 16.6 & 13.0 \\
\hline Jews & 4.4 & 5.4 & 5.3 & 5.0 & 4.6 & 2.3 \\
\hline Germans & 16.3 & 0.7 & 7.9 & 3.9 & 3.5 & 1.3 \\
\hline Others & 18.4 & 14.4 & 17.9 & 21.9 & 26.9 & 22.4 \\
\hline Total & $1,126.4$ & $1,196.8$ & $1,356.1$ & $1,464.5$ & $1,565.7$ & $1,445.6$ \\
\hline \multicolumn{7}{|c|}{ LATVIA } \\
\hline & $1935^{\mathrm{a}}$ & 1959 & 1970 & 1979 & 1989 & $1999^{b}$ \\
\hline Latvians & $1,472.6$ & $1,297.9$ & $1,341.8$ & $1,344.1$ & $1,387.8$ & $1,357.8$ \\
\hline Russians & 206.5 & 556.4 & 704.6 & 821.5 & 905.5 & 788.4 \\
\hline Belorussians & 26.9 & 61.6 & 94.9 & 111.5 & 119.7 & 95.6 \\
\hline Ukrainians & 1.8 & 29.4 & 53.5 & 66.7 & 92.1 & 70.8 \\
\hline Poles & 48.9 & 59.8 & 63.0 & 62.7 & 60.4 & 53.1 \\
\hline Lithuanians & 22.9 & 32.4 & 40.6 & 37.8 & 34.6 & 31.1 \\
\hline Jews & 93.5 & 36.6 & 36.7 & 28.3 & 22.9 & 8.6 \\
\hline Germans & 62.1 & 1.6 & 5.4 & 3.3 & 3.8 & 1.5 \\
\hline Others & 15.3 & 17.8 & 23.6 & 26.9 & 39.8 & 32.5 \\
\hline Total & $1,950.5$ & $2,093.5$ & $2,364.1$ & $2,502.8$ & $2,666.6$ & $2,439.4$ \\
\hline \multicolumn{7}{|c|}{ LITHUANIA } \\
\hline & $1923^{\mathrm{a}}$ & 1959 & 1970 & 1979 & 1989 & $1997^{b}$ \\
\hline Lithuanians & $1,739.5$ & $2,150.8$ & $2,506.8$ & $2,712.2$ & $2,924.3$ & $3,024.3$ \\
\hline Russians & 50.7 & 231.0 & 268.0 & 303.5 & 344.5 & 304.8 \\
\hline Poles & 65.6 & 230.1 & 240.2 & 247.0 & 258.0 & 256.6 \\
\hline Belorussians & 4.4 & 30.3 & 45.4 & 57.6 & 63.2 & 54.5 \\
\hline Ukrainians & - & 17.7 & 25.1 & 32.0 & 44.8 & 36.9 \\
\hline Jews & 154.3 & 24.7 & 23.6 & 14.7 & 12.4 & 5.2 \\
\hline Germans & 88.6 & 11.2 & - & - & - & - \\
\hline Others & 55.1 & 15.6 & 19.1 & 24.5 & 27.6 & 24.9 \\
\hline Total & $2,158.2$ & $2,711.4$ & $3,128.2$ & $3,391.5$ & $3,674.8$ & $3,707.2$ \\
\hline
\end{tabular}

aPrewar borders

'Figures for the late 1990s are estimates; all others are based on census data.

Sources: Egil Levits, "Die demographische Situation in der UdSSR und in den baltischen Staaten unter besonderer Berücksichtigung von nationalen und sprachsoziologischen Aspekten," Acta Baltica, 21 (1981), 63, 90, 119; Eesti arvudes 1920-1935 (Tallinn, 1937) 12; Kalev Katus, "Rahvus: sakslane; elukoht: Eesti,” Aja Pulss, no. 22 (1990) 10; Eesti statistika aastaraamat 1999 (Tallinn, 1999), 33; Latvijas statistikas gadagrāmata 1999 (Riga, 1999), 58; Lietuvos statistikos metraštis 1997 (Vilnius, 1997), 32 . 
Table 2. Ethnic composition of the Baltic States (\%)

ESTONIA

\begin{tabular}{|c|c|c|c|c|c|c|}
\hline & $1934^{\mathrm{a}}$ & 1959 & 1970 & 1979 & 1989 & $1999^{\circ}$ \\
\hline Estonians & 88.2 & 74.6 & 68.2 & 64.7 & 61.5 & 65.2 \\
\hline Russians & 8.2 & 20.1 & 24.6 & 27.9 & 30.3 & 28.1 \\
\hline Ukrainians & - & 1.3 & 2.1 & 2.5 & 3.1 & 2.5 \\
\hline Belorussians & - & 0.9 & 1.4 & 1.6 & 1.8 & 1.5 \\
\hline Finns & 0.1 & 1.4 & 1.4 & 1.2 & 1.1 & 0.9 \\
\hline Jews & 0.4 & 0.5 & 0.4 & 0.3 & 0.3 & 0.2 \\
\hline Germans & 1.5 & 0.1 & 0.6 & 0.3 & 0.2 & 0.1 \\
\hline Others & 1.6 & 1.2 & 1.3 & 1.5 & 1.7 & 1.5 \\
\hline Total $^{b}$ & 100.0 & 100.1 & 100.0 & 100.0 & 100.0 & 100.0 \\
\hline \multicolumn{7}{|c|}{ LATVIA } \\
\hline & $1935^{\mathrm{a}}$ & 1959 & 1970 & 1979 & 1989 & $1999^{\mathrm{c}}$ \\
\hline Latvians & 75.7 & 62.0 & 56.8 & 53.7 & 52.0 & 55.7 \\
\hline Russians & 10.6 & 26.6 & 29.8 & 32.8 & 34.0 & 32.3 \\
\hline Belorussians & 1.4 & 2.9 & 4.0 & 4.5 & 4.5 & 3.9 \\
\hline Ukrainians & 0.01 & 1.4 & 2.3 & 2.7 & 3.4 & 2.9 \\
\hline Poles & 2.5 & 2.9 & 2.7 & 2.5 & 2.3 & 2.2 \\
\hline Lithuanians & 1.2 & 1.5 & 1.7 & 1.5 & 1.3 & 1.3 \\
\hline Jews & 4.8 & 1.8 & 1.6 & 1.1 & 0.9 & 0.4 \\
\hline Germans & 3.2 & 0.1 & 0.2 & 0.1 & 0.1 & 0.1 \\
\hline Others & 0.8 & 0.9 & 1.0 & 1.1 & 1.5 & 1.2 \\
\hline Total $^{\mathrm{b}}$ & 100.2 & 100.1 & 100.1 & 100.0 & 100.0 & 100.0 \\
\hline \multicolumn{7}{|c|}{ LITHUANIA } \\
\hline & $1923^{\mathrm{a}}$ & 1959 & 1970 & 1979 & 1989 & $1997^{c}$ \\
\hline Lithuanians & 80.6 & 79.3 & 80.1 & 80.0 & 79.6 & 81.6 \\
\hline Russians & 2.3 & 8.5 & 8.6 & 8.9 & 9.4 & 8.2 \\
\hline Poles & 3.0 & 8.5 & 7.7 & 7.3 & 7.0 & 6.9 \\
\hline Belorussians & 0.2 & 1.1 & 1.5 & 1.7 & 1.7 & 1.5 \\
\hline Ukrainians & - & 0.7 & 0.8 & 0.9 & 1.2 & 1.0 \\
\hline Jews & 7.2 & 0.9 & 0.8 & 0.4 & 0.3 & 0.1 \\
\hline Germans & 4.1 & 0.4 & - & - & - & - \\
\hline Others & 2.6 & 0.6 & 0.6 & 0.7 & 0.8 & 0.7 \\
\hline Total $^{\mathrm{b}}$ & 100.0 & 100.0 & 100.1 & 99.9 & 100.0 & 100.0 \\
\hline
\end{tabular}

${ }^{\text {a }}$ Prewar borders

${ }^{\mathrm{b}}$ Due to rounding off totals are not always $100.0 \%$

${ }^{c}$ Figures for the late 1990s are estimates; all others are based on census data.

Sources: Egil Levits, "Die demographische Situation in der UdSSR und in den baltischen Staaten unter besonderer Berücksichtigung von nationalen und sprachsoziologischen Aspekten," Acta Baltica, 21 (1981), 64, 91, 120; Eesti arvudes 1920-1935 (Tallinn, 1937), 12; Kalev Katus, "Rahvus: sakslane; elukoht: Eesti," Aja Pulss, no. 22 (1990), 10; Eesti statistika aastaraamat 1999 (Tallinn, 1999), 33; Latvijas statistikas gadagrāmata 1999 (Riga, 1999), 58; Lietuvos statistikos metraštis 1997 (Vilnius, 1997), 32 . 
